A major purpose or the I ecnnical Information Center is to provide the broadest dissemination possible of information contained in DOE's Research and Development Reports to business, industry, the academic community', and federal, state and local governments.

Although a small portion of this report is not reproducible, it is being made available to expedite the availability of information on the research discussed herein. 
Airtio o 1989

TITLE:

$\operatorname{AUTHOR}(5)$ :

SUBMITTED TO:

\section{PHOTOELECTRIC INJECTOR DESIGN CODE}

LA-UR- $-89-935$

DE89 009282

\title{
DISCI.AIMER
}

\begin{abstract}
This repurt was prepared as an account of work spunsored by an agency of the l/nited Situtes fovernment Neither the l Inited Slates Covernment nor any agency thereol. nor ans wh their emploves, makes ant warrants. express or implied. of assumes anv iegal habilit ur responst

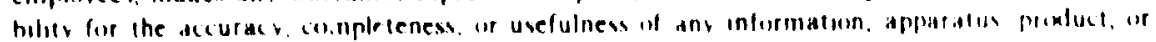

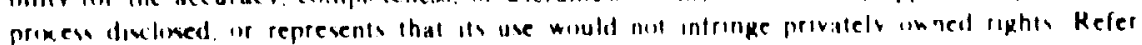

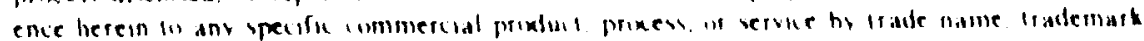

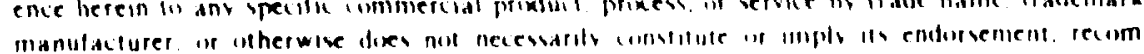

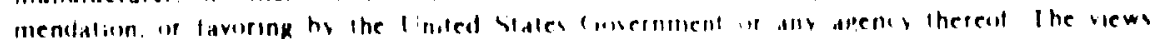

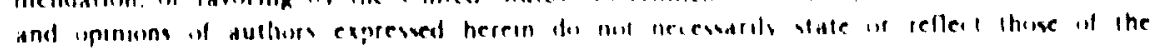
l'nited siates fovernment or ans akens, thesed
\end{abstract}

1989 Particle Acceierator Conference

Chicago, IL

March 20-23, 1989

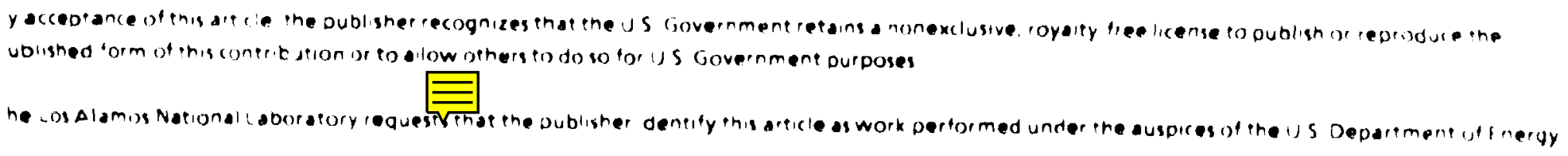

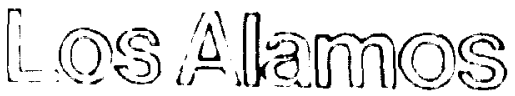

Los Alamos Nafional Laboralor

Los Alamos New Mexico 8754 .5 
Bruce F. C'arlsten

MS-Hk25, Los Alamos National Laboratory, Los Alamos, NM N75.45

\section{Abstract}

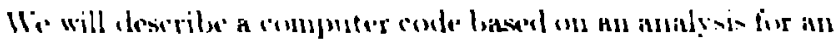

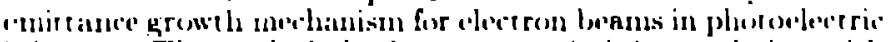
injertors. The analysis leiuls to a generio injector design with

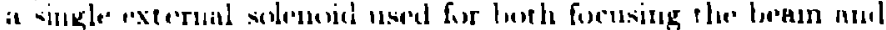

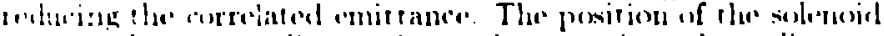

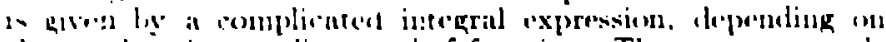

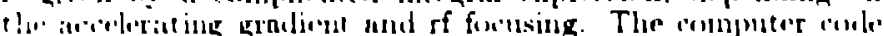

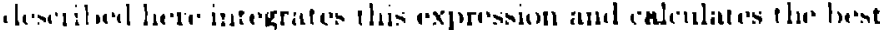

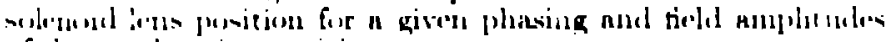
of the acrolerating cavit ies.

\section{Int roduction}

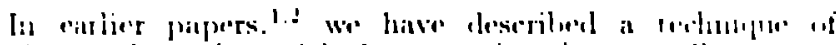

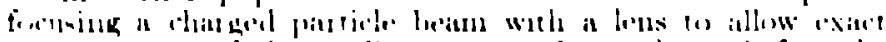

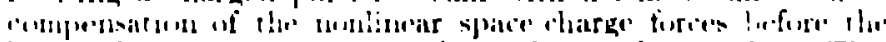

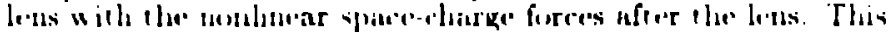

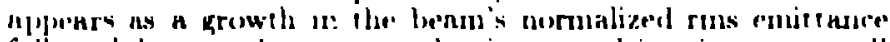

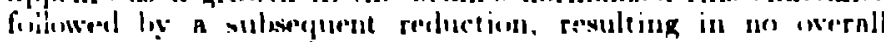

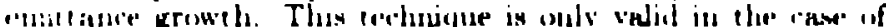

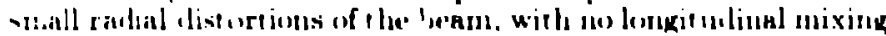

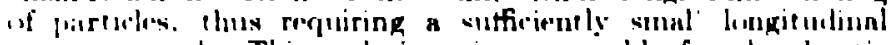

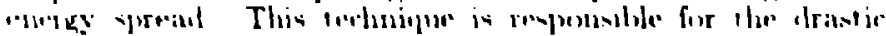

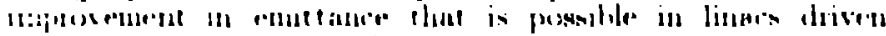

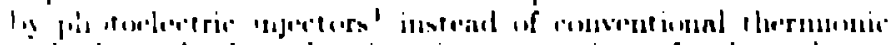

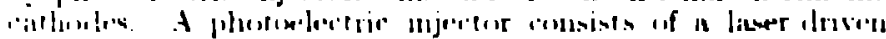

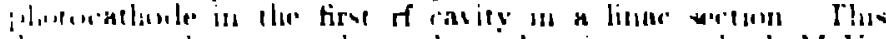

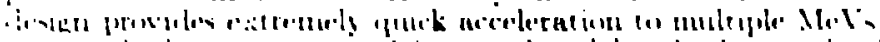

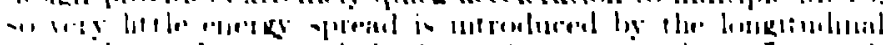

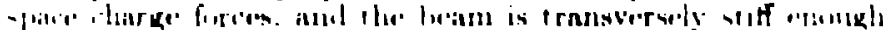

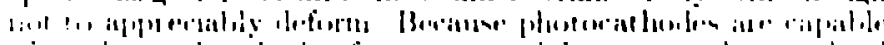

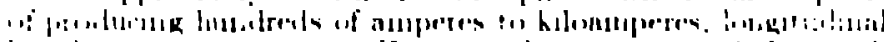

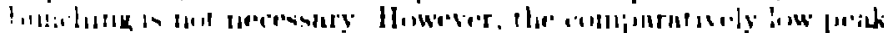

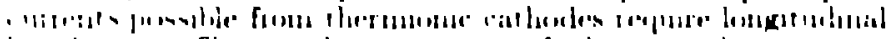

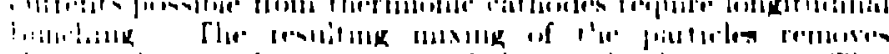

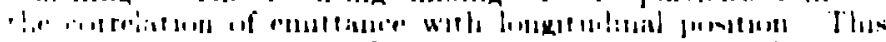

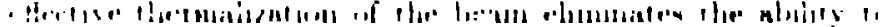

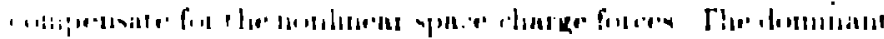

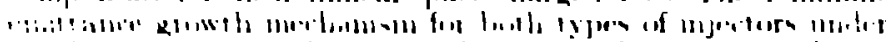

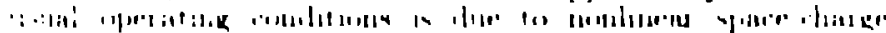

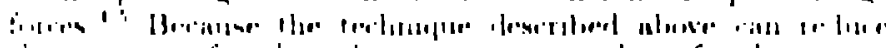

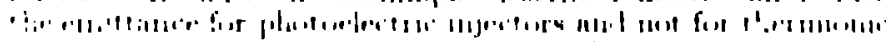

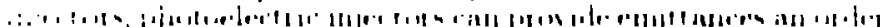

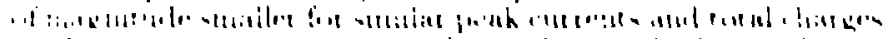

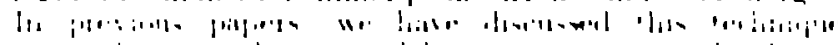

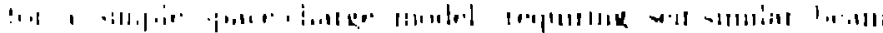

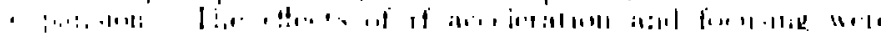

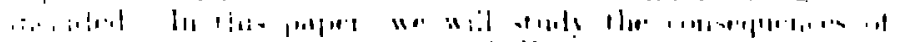

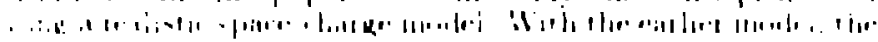

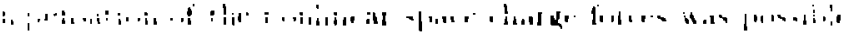

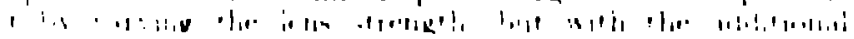

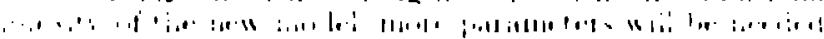

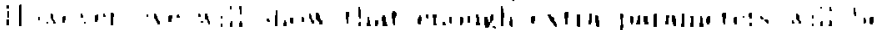

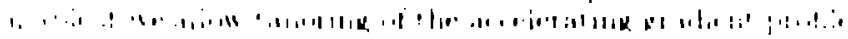

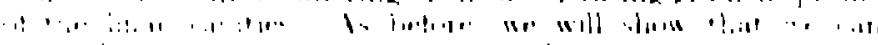

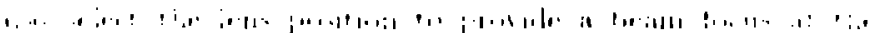

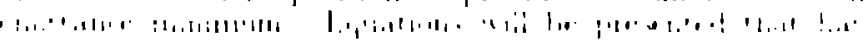

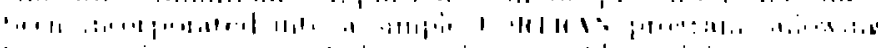

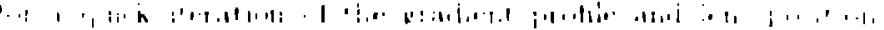

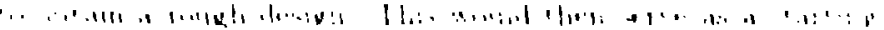

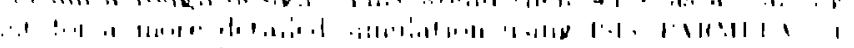

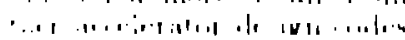

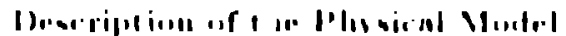

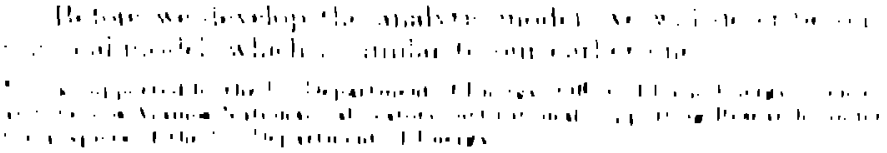




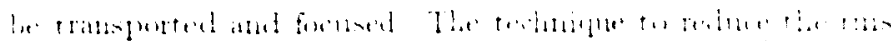

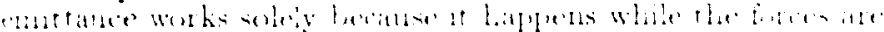
still monlinear.

\section{Analytic Model}

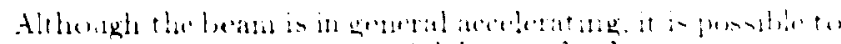

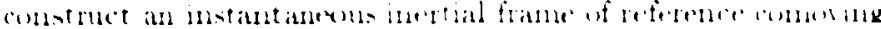

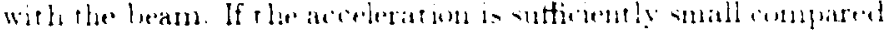

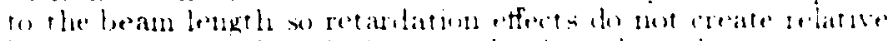

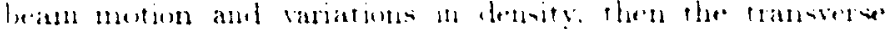

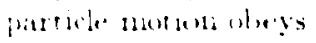

$$
\frac{i 2 r}{i+\frac{1}{n}}=(n+\cdots)
$$

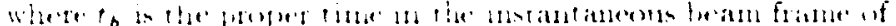

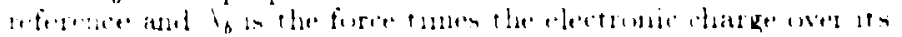

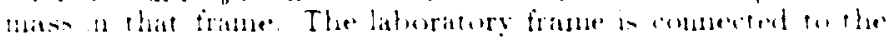
Heatli frame la

$$
-x_{1} \cdot t_{h}=d z
$$

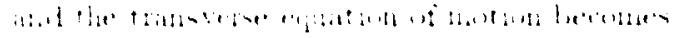

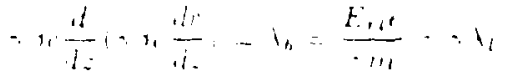

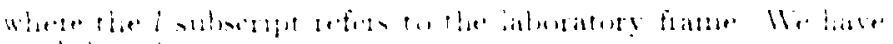

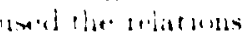

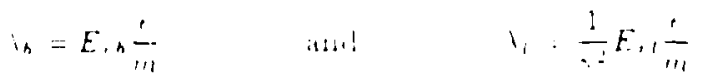

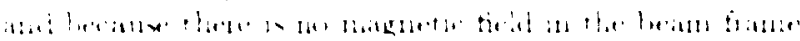

$$
E_{1,} \cdot F_{, n}
$$

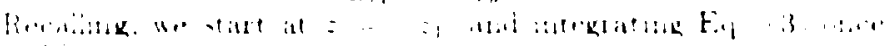

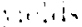

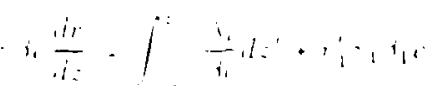

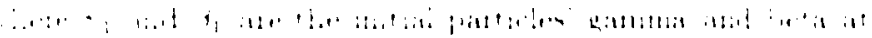

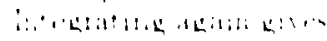

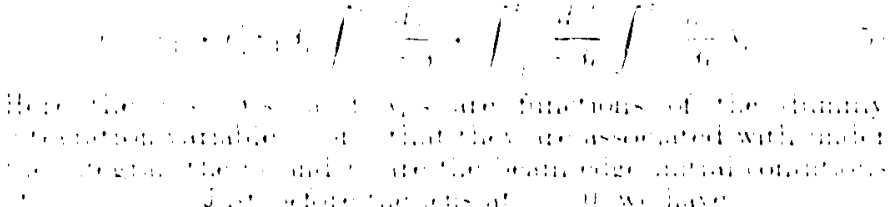

$$
\begin{aligned}
& \cdots \quad j \quad j \quad ; j
\end{aligned}
$$$$
\therefore:
$$$$
\therefore \quad, \quad, \quad \vdots \quad \ldots
$$$$
, \ldots, \ldots, \ldots, \ldots, 13, \ldots, \ldots, \ldots, \ldots, \ldots
$$

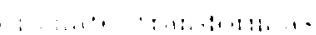

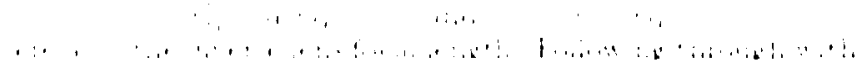

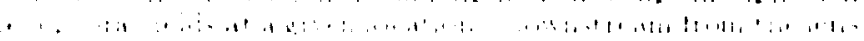

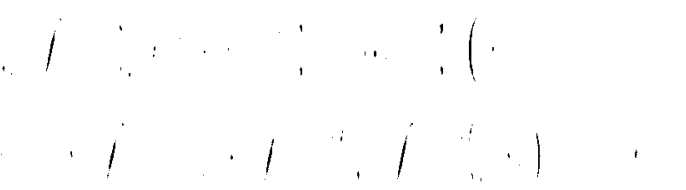

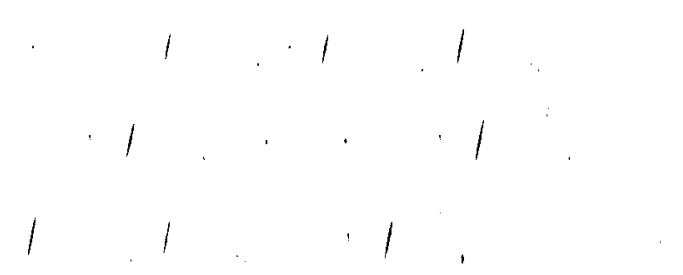

Wha

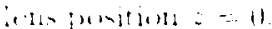

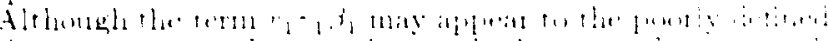

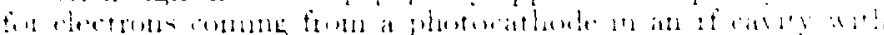

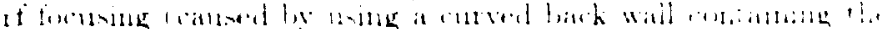

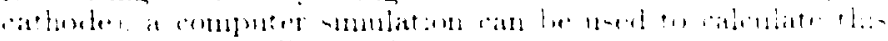
tom whls only shlall ertor trum

$$
\therefore i_{1}=-\frac{1}{1 \div \frac{1}{1}}
$$

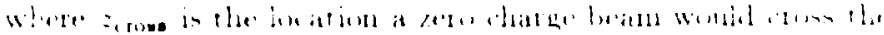
axis

\section{Space-Charge Model}

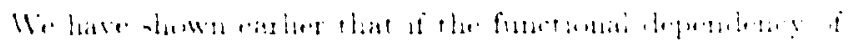

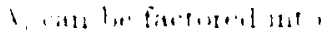

$$
1,2, \therefore i=
$$

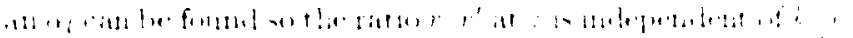

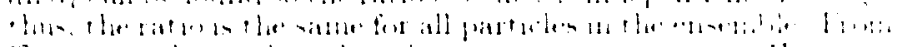

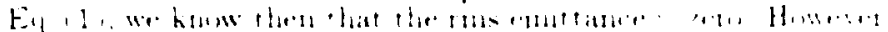

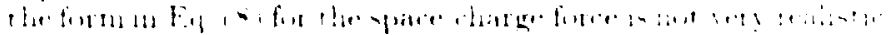

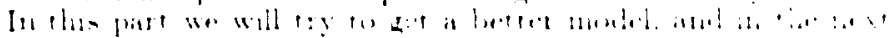

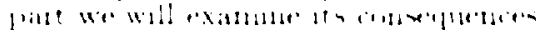

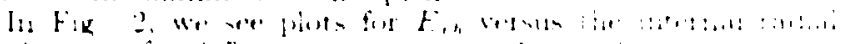

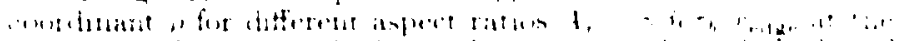

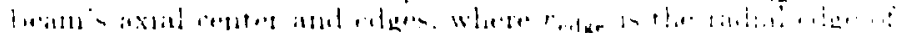

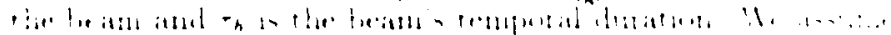

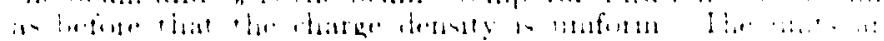

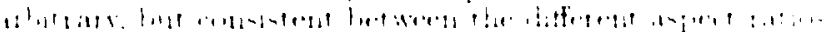

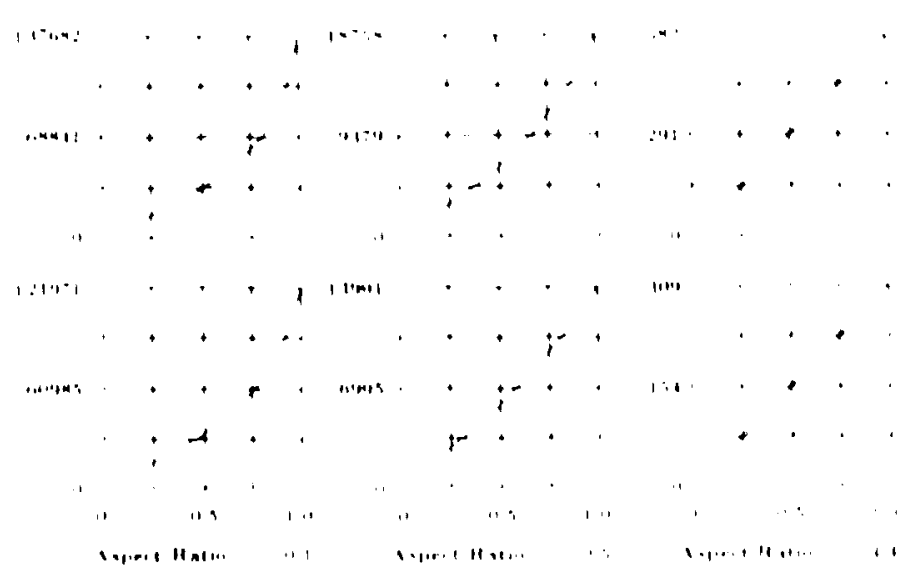

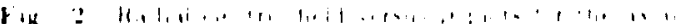

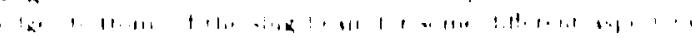

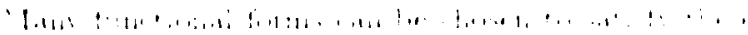
14

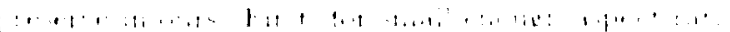

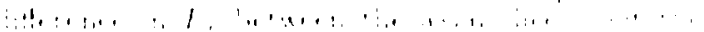

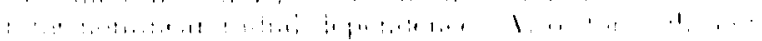

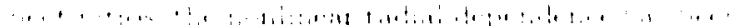

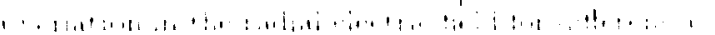
" \& \&

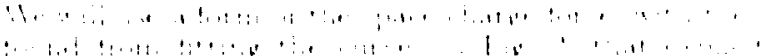

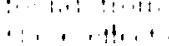




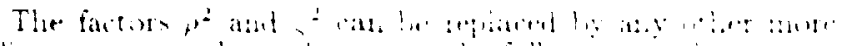

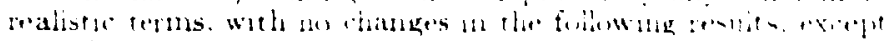

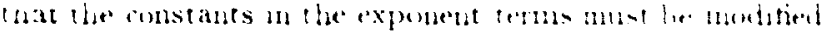

\section{Integral Equations}

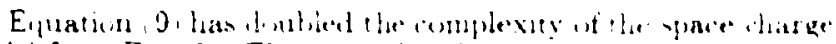

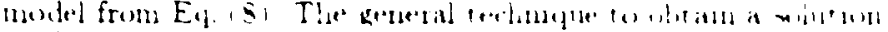
whint

$$
\frac{r_{1}}{r_{1}}=\frac{r_{1}}{r^{\prime}}
$$

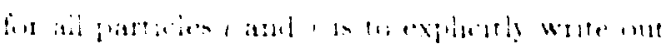

$$
11=r, r ;, r, r
$$

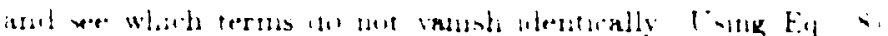

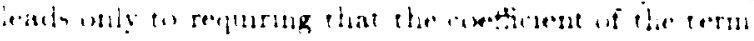

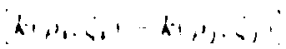

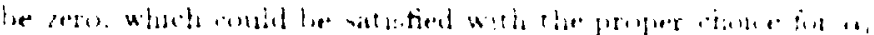

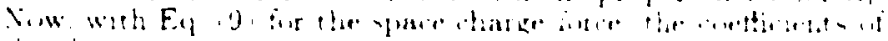

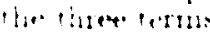

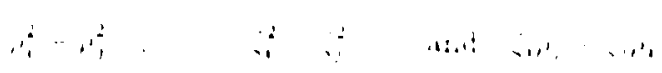

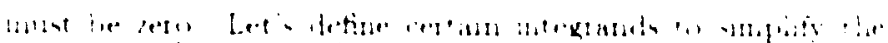

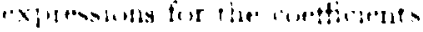

$$
\begin{aligned}
& i: \quad, \\
& \text { l. } 1.228 \text { t. 1. } \\
& \text { I } \frac{1}{1}(1,1 . \cdots)
\end{aligned}
$$

X... N. N!!

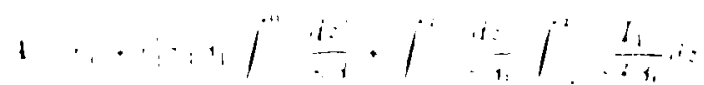

$$
\begin{aligned}
& +j^{i} i, \quad \therefore
\end{aligned}
$$

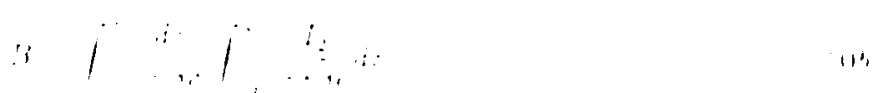

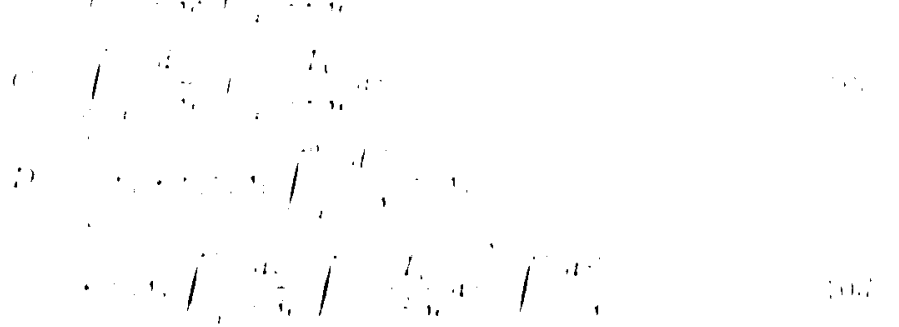

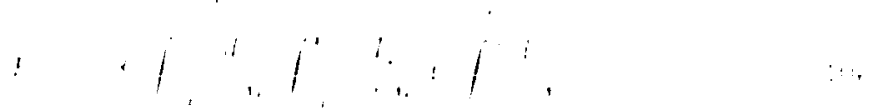

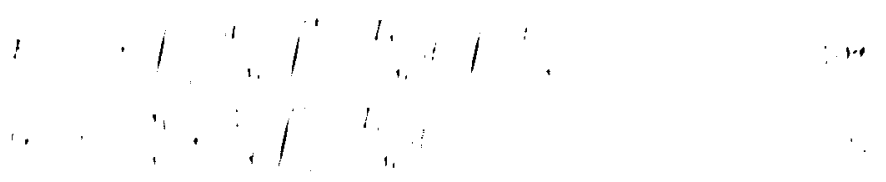

$$
\begin{aligned}
& \begin{array}{llll}
11 & 1 & 1 & 1 \\
1 & 1 & 1 & 1 \\
1 & 1 & 1 & 1
\end{array}
\end{aligned}
$$

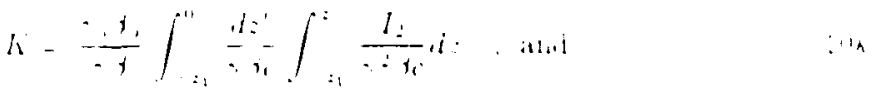

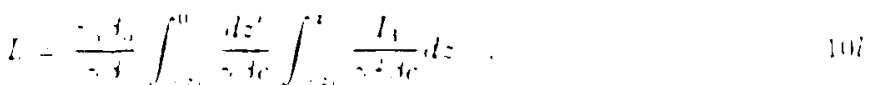

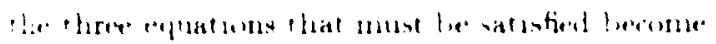

$$
\begin{aligned}
& (B I-(H) \quad, H E I \cdot B L-C K \cdot F H: \|) \therefore
\end{aligned}
$$

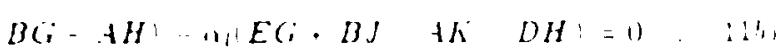

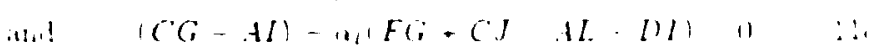

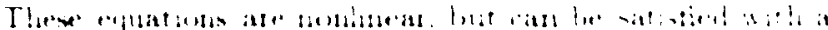

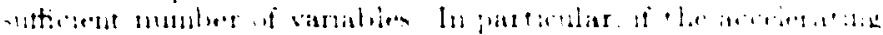

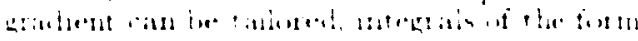

$$
\int \frac{d z}{i} \quad \ln 1 \frac{i}{2}-1
$$

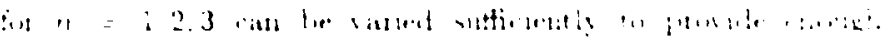

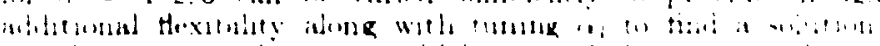

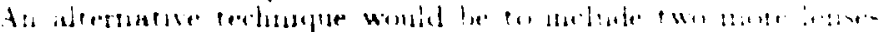

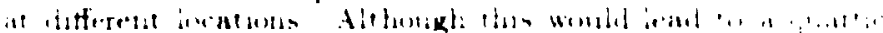

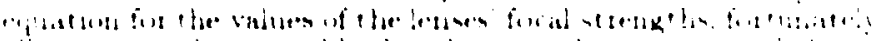

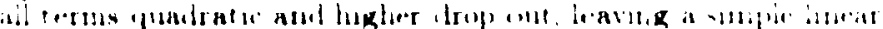

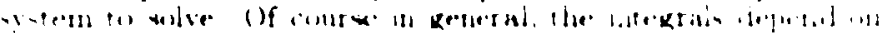

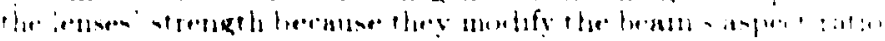

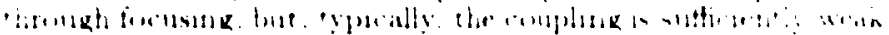

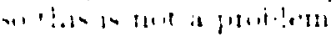

\section{IParation Procedure}

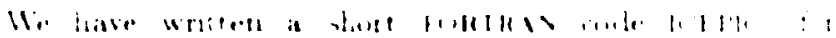

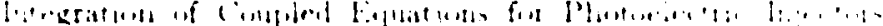

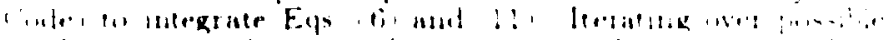

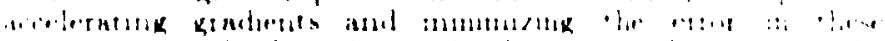

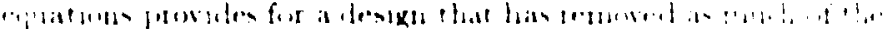

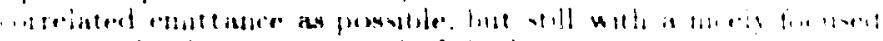

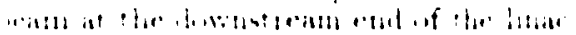

\section{('omelunion)}

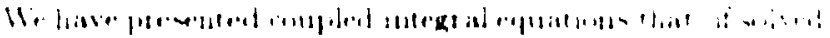

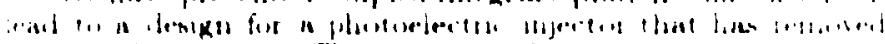

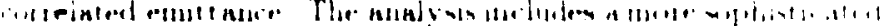

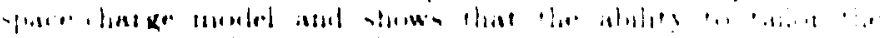

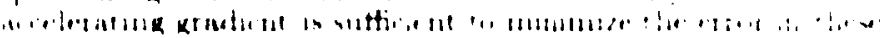

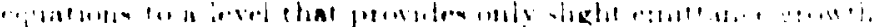

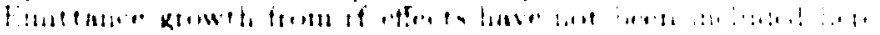

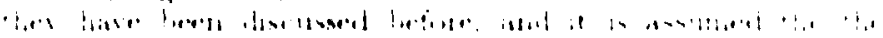

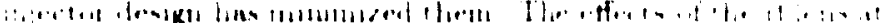

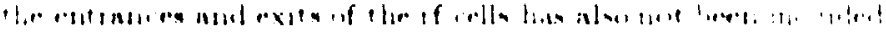

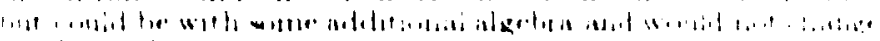

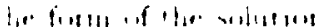

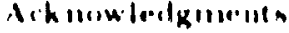

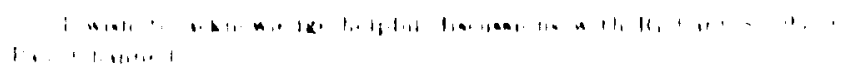

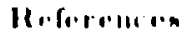

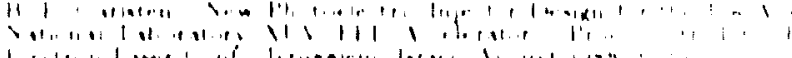

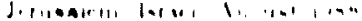

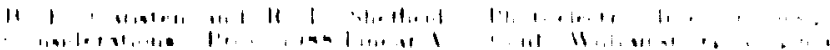

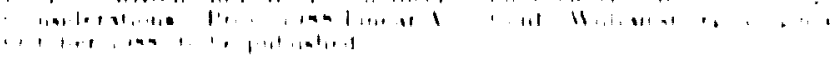

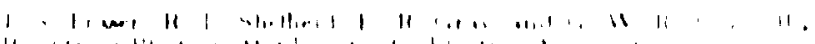

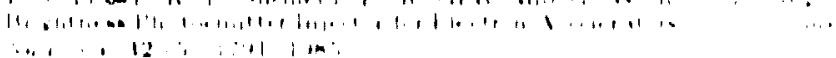

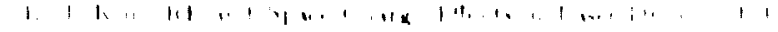

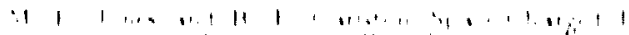

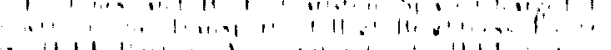

